\title{
Bemerkungen.
}

Nov. 26. Comet schwach, verwaschen; etwa o.'5 Durchmesser, doch gut an hellen Fäden zu beobachten. - Dec. 2. Die Nähe des Vergleichsterns störte die Auffassung des Cometen, daher wurden die Beobachtungen bald abgebrochen; $\Delta \delta$ sehr unsicher. - Dec. 3. Comet recht gut zu sehen, etwa I' Durchmesser. - Dec. 4. Die unmittelbare Nähe eines Sterns $\mathrm{I}^{\mathrm{m}}$ störte die Beobachtung. - Dec. 8. Comet trotz Mondscheins und leichter Cirrostratus gut zu sehen, verwaschen, über 2' Durchmesser, rund, central verdichtet.

Die Helligkeit war durchschnittlich die eines Sterns $\mathrm{II}^{\mathrm{m}}-\mathrm{r} 2^{\mathrm{tn}}$, am 26 . Nov. nahe an $\mathrm{II}^{\mathrm{m}}$, am 4. Dec. geringer als $\mathrm{I}^{1} 1^{1 / 2}$, am 8. Dec. $\mathrm{I}^{\mathrm{m}}$. Vergr. 60 f., am 26. Nov. $80 \mathrm{f}$.

Jena II, I896 Dec. 9.

W. Winkler.

\section{Ephemeride für den periodischen Cometen Brooks (1889 V) 1896 VI.}

Fortsetzung zu A. N. 3389.

$12^{\text {h }}$ M. Z. Berlin.

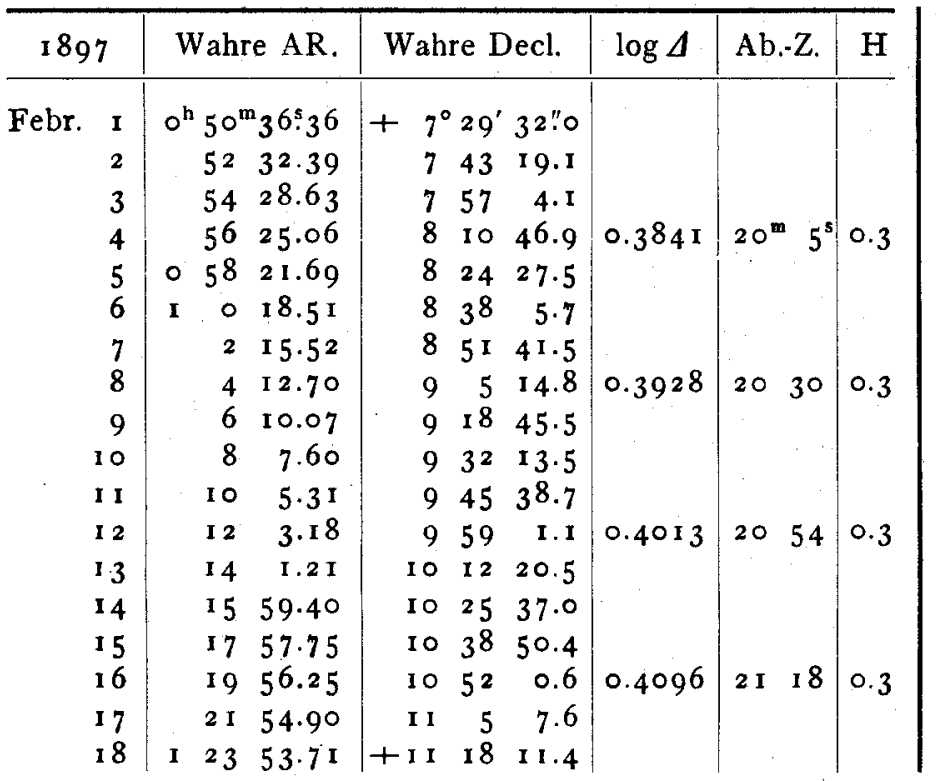

Berlin 1897 Jan. 27.

\begin{tabular}{|c|c|c|c|c|c|c|c|}
\hline \multicolumn{2}{|c|}{1897} & Wahre AR. & \multicolumn{2}{|c|}{ Wahre Decl. } & $\log A$ & Ab. $-Z$. & $\mathrm{H}$ \\
\hline Febr. & & $1^{h} 25^{m} 5^{2^{s}} \cdot 67$ & $+11^{\circ} 31^{\prime}$ & I I":8 & & & \\
\hline & 20 & 2751.77 & I I 44 & 8.8 & 0.4177 & $21^{\mathrm{m}} 42^{\mathrm{s}}$ & 0.3 \\
\hline & $2 \mathbf{I}$ & 2951.02 & I 57 & 2.4 & & & \\
\hline & 22 & 3 I 50.43 & I 29 & $5^{2 \cdot 5}$ & & & \\
\hline & 23 & $33 \quad 49.97$ & 1222 & 39.0 & & & \\
\hline & 24 & $35 \quad 49.67$ & 1235 & 21.9 & 0.4255 & 22 & 0.3 \\
\hline & 25 & 3749.52 & 1248 & I. I & & & \\
\hline & 26 & 3949.51 & I 30 & $3^{6.6}$ & & & \\
\hline & 27 & $41 \quad 49.65$ & I 3 I 3 & 8.3 & & & \\
\hline & 28 & $43 \quad 49.92$ & I 325 & 36.2 & $0.433^{2}$ & 2230 & 0.3 \\
\hline März & $\mathbf{I}$ & $45 \quad 50.34$ & 1338 & 0.0 & & & \\
\hline & 2 & $47 \quad 50.89$ & I 350 & 19.9 & & & \\
\hline & 3 & 4951.58 & I 42 & $35 \cdot 7$ & & & \\
\hline & 4 & $\begin{array}{ll}51 & 52.39\end{array}$ & 1414 & $47 \cdot 4$ & 0.4407 & 2253 & 0.2 \\
\hline & 5 & $53 \quad 53.33$ & 1426 & 54.9 & & & \\
\hline & 6 & $55 \quad 54.40$ & $143^{8}$ & 58.1 & & & \\
\hline & 7 & $57 \quad 55.59$ & 1450 & $5^{6.9}$ & & & \\
\hline & 8 & I $59 \quad 56.90$ & +152 & 51.4 & 0.4480 & $23 \quad 16$ & 0.2 \\
\hline
\end{tabular}

F. Bauschinger.

Neue Elemente des Cometen 1896 ...(Perrine Dec. 8).

Die Elemente beruhen auf drei Normalörtern 1896 Dec. I 0.5 (7 Beob.), I 896 Dec. 28.5 (6 Beob) und 1897 Jan. 5.5 (I 4 Beob.), zu denen ich die mir bekannt gewordenen Beobachtungen zusammengezogen habe. Dieselben stellen eine Nizzaer Beobachtung vom I2. Januar bis auf $\Delta \alpha=+0.18, \Delta \delta=-0.3$ dar.

Die Elemente lauten:

$$
\begin{aligned}
& T=1896 \text { Nov. } 24.65674 \text { M. Z. B. } \\
& \omega=163^{\circ} 53^{\prime} 30^{\prime \prime} \circ \\
& \left.\delta=24^{6} 34 \quad 35.9\right\} \quad \text { I } 897.0 \\
& i=134025.9 \\
& \varphi=424717.0 \\
& \log e=9.832054 \\
& \log q=0.045405 \\
& \mu=550.901 \\
& a=3.4617 \\
& U=6.44 I \text { Jahre }
\end{aligned}
$$

Heliocentrische Aequatorealcoordinaten.

$$
\begin{aligned}
& x=\left[9.9^{8} 9534^{6}\right] r \cdot \sin \left(v+139^{\circ} 5 \mathrm{I}^{\prime} 4 \mathrm{I}^{\prime \prime} 40\right) \\
& y=[9.9794489] r \cdot \sin (z+455049.2 \mathrm{I}) \\
& z=[9.5689189] r \cdot \sin (v+8342 \text { 3.13) }
\end{aligned}
$$

Der Comet nimmt in den heliocentrischen Längen $216^{\circ}$ und $247^{\circ}$ dieselben Radienvectoren an, die dort der Jupitersbahn zugehören, das erste Mal $30^{1 / 2}$ Grad vor dem aufsteigenden Knoten, das zweite Mal nur 1/2 Grad nach dem Durchgang durch den aufsteigenden Knoten. Hier kann die grösste Annäherung an Jupiter bis auf 0.053 stattfinden. Seit 1852 ist das zwar nicht eingetreten - da der Comet dann in kleinerer nördlicher heliocentrischer Breite dicht unter Jupiter durchgeht, so wäre eine starke Aenderung der Neigung, also eine viel schlechtere Uebereinstimmung der Elemente mit denen des Cometen Biela das Resultat -, wohl aber haben im Jahre 1888 grosse Jupiterstörungen stattgefunden. Als damals am 27. April der Comet durch 